\title{
SOME COMBINATORIAL THEOREMS EQUIVALENT TO THE PRIME IDEAL THEOREM
}

R. H. COWEN

Abstract. Some useful combinatorial selection lemmas are shown to be directly equivalent to the prime ideal theorem for boolean algebras.

1. The theorems we shall consider are intimately related to R. Rado's selection lemma (Theorem 2, below) which first appeared in [10] and subsequently has found wide application (see [1], [3], [4], [12]). Our main theorem is Theorem 1 which we use to derive other forms of Rado's lemma and to prove A. Robinson's valuation lemma which was shown by Robinson in [9] to be a fundamental result in model theory.

We also show that Theorem 1 and some of the theorems we derive from it are equivalent to the prime ideal theorem for boolean algebras and thus we have some useful abstract versions of this theorem, versions divorced from any particular algebraic structure. Whether the original lemma of Rado is as strong as the prime ideal theorem appears to be an open question; note, however, that E. S. Wolk [12] has shown that Rado's lemma plus the axiom of choice for families of finite sets implies the Tychonoff theorem for finite spaces, which, in turn, implies the prime ideal theorem (see [8]). We conjecture that Rado's lemma is strictly weaker than the prime ideal theorem.

2. Preliminaries. In this paper we shall work, informally, within the framework of Zermelo-Fraenkel set theory (ZF). All uses of the axiom of choice will be explicitly noted.

Given a set $I$, by a partial function on $I$, we mean a function whose domain is a subset of $I$. A partial valuation on $I$ is a partial function on $I$ whose range is included in $\{0,1\}$. As in $\mathrm{ZF}$ we consider functions to be special sets of ordered pairs and we even allow $\varnothing$, the empty function (the empty set of ordered pairs). If $f$ is a partial function on $I$ we write $D(f)$ for its domain, and if $U \subseteq I$ we write $f \nmid U$ for the restriction of $f$ to $U \cap D(f)$.

Presented to the Society, August 30, 1972; received by the editors November 28, 1972.

AMS (MOS) subject classifications (1970). Primary 02K99, 05 A05.

Key words and phrases. Rado's lemma, selection lemma, valuation lemma, prime ideal theorem.

(c) American Mathematical Society 1973 
A set $X$ is of finite character just in case $x$ is in $X$ if and only if every finite subset of $x$ is in $X$. It is trivial so show that if $X$ is of finite character, then $y \subseteq x \in X$ implies $y \in X$. The Tukey-Teichmüller principle, a form of the axiom of choice, states that every nonempty set of finite character has a maximal element under inclusion (see [5, p. 33]). If $F$ is a set of partial functions on $I$, then $F$ is of finite character if and only if: $f \in F$ if and only if $f\lceil W \in F$ for every finite $W \subseteq I$.

Finally, $N$, a nonempty set of subsets of $I$, is a net on $I$ if $J_{1}, J_{2} \in N$ implies there exists $J_{3} \in N$ such that $J_{3} \subseteq J_{1} \cap J_{2}$.

3. Main results. In this section we state and prove Theorem 1 and derive several consequences. The proof we give here of Theorem 1 is essentially the same as our proof of Rado's lemma in [2].

THEOREM 1. Suppose for each finite $W \subseteq I$ there is a nonempty set $H_{W}$ of partial functions on I whose domains include $W$ and such that $W_{1} \subseteq W_{2}$ implies $H_{W_{2}} \subseteq H_{W_{1}}$. Suppose also that, for each $v \in I,\left\{h(v) \mid h \in H_{\varnothing}\right\}$ is a finite set. Then there exists a function $g$, with domain I, such that for any finite $W$ there exists $h \in H_{W}$ with $g \backslash W \subseteq h$.

Proof. First we note that since $W_{1} \subseteq W_{2}$ implies $H_{W_{2}} \subseteq H_{W_{1}}, H_{\varnothing}=$ $\bigcup H_{W}$, the union being taken over all finite $W \subseteq I$.

Let $F$ be the set of all partial functions $f$ on $I$ such that for any finite $W \subseteq I$, there is an $h \in H_{W}$ such that $f \nmid W \subseteq h$. We will show there is a function in $F$ with domain $I$.

$F$ is not empty; in fact $\varnothing \in F$.

Next we show $F$ is of finite character. Suppose $f \in F$ and $V$ is a finite subset of $I$. Given any finite $W \subseteq I$, there is an $h \in H_{W}$ such that $f \backslash W \subseteq h$, so certainly $(f \backslash V) \mid W \subseteq h$. This shows $f \backslash V \in F$. Conversely if $f \backslash V \in F$ for all finite $V \subseteq I$, then, for any finite $W \subseteq I, f \backslash W \in F$, so there is an $h \in H_{W}$ such that $(f \backslash W)\lceil W \subseteq h$, that is, $f \backslash W \subseteq h$. Therefore $f \in F$.

The Tukey-Teichmüller principle gives us a maximal $g$ in $F$. We claim $D(g)=I$. Suppose not; if $v \in I-D(g)$, let $\left\{h(v) \mid h \in H_{\varnothing}\right\}=\left\{a_{1}, \cdots, a_{n}\right\}$ and let $g_{i}=g \cup\left\{\left\langle\nu, a_{i}\right\rangle\right\}, 1 \leqq i \leqq n$. Since $g$ is maximal in $F, g_{i} \notin F$ and so there exists $W_{i}$ such that $\left.g_{i}\right\rceil W_{i} \subseteq h$ is false for all $h \in H_{W_{i}}$. We will show this is impossible. Surely $v \in W_{i}$, since otherwise $g \uparrow W_{i}=g_{i} \uparrow W_{i}$ and then $g \notin F$ ! Let $W=\bigcup_{i=1}^{n} W_{i}$. Since $W$ is finite and $g \in F$, there exists $h \in H_{W}$ such that $g \nmid W \subseteq h$. Therefore $g \nmid W_{i} \subseteq g \nmid W \subseteq h, 1 \leqq i \leqq n$. Surely $v \in W \subseteq$ $D(h)$, so $h(v)=a_{j}$, for some $j, 1 \leqq j \leqq n$. Then $g_{j} \uparrow W_{j}=g \nmid W_{j} \cup\left\{\left\langle v, a_{j}\right\rangle\right\} \subseteq h$. Also, since $W_{j} \subseteq W, H_{W} \subseteq H_{W_{j}}$; therefore $h \in H_{W_{j}}$, which together with $g_{j} \uparrow W_{j} \subseteq h$ is a contradiction. Hence $D(g)=I$.

Theorem 2 (R. RADo). Let $\left\{A_{v} \mid v \in I\right\}$ be a set of finite sets. Suppose for every finite $W \subseteq I$ there exists a function $f_{W}, D\left(f_{W}\right)=W$, such that 
$f_{W}(v) \in A_{v}, v \in W$. Then there is a function $f, D(f)=I$, such that for every finite $W \subseteq I$ there exists a finite $W^{\prime}, W \subseteq W^{\prime} \subseteq I$ with $f(v)=f_{W^{\prime}}(v)$, for all $v \in W$.

Proof. For each finite $W \subseteq I$, let $H_{W}$ consist of all $f_{W^{\prime}}$, where $W \subseteq$ $W^{\prime} \subseteq I, W^{\prime}$ finite. Theorem 2 now follows directly from Theorem 1 .

The next theorem often gives quicker proofs of results than Rado's lemma or Theorem 1.

THEOREM 3. Let $F$ be a class of partial functions on I of finite character satisfying:

(a) $\{f(v) \mid f \in F\}$ is finite for each $v \in I$,

(b) for each finite $W \subseteq I$, there exists $f \in F$ with $D(f)=W$.

Then $F$ contains a function with domain $I$.

Proof. For each finite $W \subseteq I$, let $H_{W}$ consist of those functions in $F$ whose domains include $W$. It is easy to check that the hypothesis of Theorem 1 holds; the conclusion gives a function $g, D(g)=I$, such that for any finite $W$ there exists $h \in H_{W} \subseteq F$ with $g \nmid W \subseteq h$. Since $h \in F$ and $F$ is of finite character, $g \uparrow W \in F$; hence $g \in F$.

The next result, the valuation lemma of [9] and its corollary, the special valuation lemma, are employed by Robinson to give rather direct proofs of theorems on ultraproducts and the completeness theorem for first order logic, respectively.

THEOREM 4 (A. RoBinson). Let $\Phi=\left\{\phi_{t}\right\}_{t \in T}$ be a set of partial valuations on a set $S$. Let $N$ be a net on $T$ such that for every finite $U \subseteq S$ and every $J \in N$ there exists $a v \in J$ for which $U \subseteq D\left(\phi_{v}\right)$. Then there exists a valuation $\Psi, D(\Psi)=S$, such that for every finite $U \subseteq S$ and every $J \in N$ there exists a $v \in J$ such that $U \subseteq D\left(\phi_{v}\right)$ and $\Psi\left\lceil U=\phi_{v} \mid U\right.$.

Proof. Let $I=S \cup N$ and for each finite $W \subseteq I$ with $W=U \cup\left\{J_{1}, \cdots\right.$, $\left.J_{n}\right\}$ where $U \subseteq S$ and $J_{i} \in N$, let $H_{W}$ consist of all partial valuations $h$ on $I$ whose domains include $W$ and such that $h \uparrow U \subseteq \phi_{v}$, for some $v \in J_{1} \cap \cdots \cap$ $J_{n}$ (if there are no $J$ 's in $W$, we interpret the intersection to be $\bigcup N$ ). $H_{W}$ is nonempty; since if $J \subseteq J_{1} \cap \cdots \cap J_{n}$ and if $v \in J$ such that $U \subseteq D\left(\phi_{v}\right)$, define $h \in H_{W}$ on $D\left(\phi_{v}\right) \cup N$ as follows: $h$ agrees with $\phi_{v}$ on $D\left(\phi_{v}\right)$ and is 0 on $N$. Also $\left\{h(v) \mid h \in H_{\varnothing}\right\}$ is finite and it is easy to check that $W_{1} \subseteq W_{2}$ implies $H_{W_{2}} \subseteq H_{W_{1}}$.

Now let $g$ be a function on $I$ satisfying the conclusion of Theorem 1 and let $\Psi=g\lceil S . D(\Psi)=S$. Given finite $U \subseteq S$ and $J \in N$, let $W=U \cup\{J\}$. Then if $h \in H_{W}$ with $g \nmid W \subseteq h$, we have $\Psi \uparrow U=g \mid U \subseteq h \uparrow U \subseteq \phi_{v}$, for some $v \in J$. Hence $U \subseteq D\left(\phi_{v}\right)$ and $\Psi \uparrow U=\phi_{v}\lceil U$ as required. 
If we let $N=\{\boldsymbol{T}\}$ in the preceding theorem we immediately obtain

THEOREM 5 (A. RoBINSON). Let $\Phi=\left(\phi_{t}\right)_{t \in T}$ be a set of partial valuations on a set $S$ such that for every finite $U \subseteq S$ there exists a $t \in T$ for which $U \subseteq D\left(\phi_{t}\right)$. Then there exists a valuation $\Psi, D(\Psi)=S$, such that for every finite $U \subseteq S$ there exists a $t \in T$ with $U \subseteq D\left(\phi_{t}\right)$ and $\Psi \uparrow U=\phi_{t} \uparrow U$.

4. We now show that all the theorems of the previous section, with the possible exception of Theorem 2 (Rado's selection lemma), are equivalent to the prime ideal theorem (in $\mathrm{ZF}$, without the axiom of choice). In Robinson [9], Theorem 5 is shown to imply the generalized completeness theorem for predicate logic which is equivalent to the prime ideal theorem (see Mendelson [7, p. 100]). In addition, we have shown that Theorem $1 \Rightarrow$ Theorem $4 \Rightarrow$ Theorem 5 . We show next Theorem $3 \Rightarrow$ Theorem 5 which will complete the proof that Theorems $1,3,4$, and 5 are at least as strong as the prime ideal theorem.

Proof that Theorem $3 \Rightarrow$ Theorem 5 . Let $F$ consist of all partial valuations $f$ on $S$ such that for every finite $U \subseteq D(f)$ there exists a $t \in T$ such that $U \subseteq D\left(\phi_{t}\right)$ and $f \backslash U \subseteq \phi_{t}$. It is sufficient to show that there is a function in $F$ whose domain equals $S . F$ is of finite character; since if $f \in F$, surely $f \nmid V \in F$ for every finite $V \subseteq S$; while if $f \nmid V \in F$ for all finite $V \subseteq S$, then, given any finite $U \subseteq D(f), f \nmid U \in F$ and $U \subseteq D(f \nmid U)$ and so there exists a $t \in T$ such that $U \subseteq D\left(\phi_{t}\right)$ and $\left(f\lceil U) \mid U \subseteq \phi_{t}\right.$, that is, $f\left\lceil U \subseteq \phi_{t}\right.$. Since $F$ consists of partial valuations, condition (a) of Theorem 3 holds; also, condition (b) holds since $\phi_{t} \in F$ for every $t \in T$. Hence there is a function in $F$ with domain $S$, as required.

We note that it is also possible to derive the prime ideal theorem directly from any of Theorems 1, 3, 4 or 5 using the facts that the existence of a prime ideal of a boolean algebra $B$ is equivalent to the existence of a 2-valued homomorphism on $B$ and that the axiom of choice is not needed to show that any finite boolean algebra contains prime ideals.

We show next that the prime ideal theorem implies Theorem 1 and hence also Theorems 3, 4, and 5. Our first proof of Theorem 1 cannot be used for this purpose since it makes use of the axiom of choice in the form of the Tukey-Teichmüller principle. We shall next give a proof of Theorem 1 from the compactness theorem for propositional logic, a consequence of the generalized completeness theorem for predicate logic which, as we have mentioned, equals the prime ideal theorem. The proof we give was suggested to the author by $\mathrm{E}$. Mendelson.

COMPACTNESS THEOREM $\Rightarrow$ THEOREM 1 .

Proof. For any finite $W \subseteq I$, let $B_{W}=\left\{h(v) \mid h \in H_{W}, v \in D(h)\right\}$. We construct the following propositional calculus: for each $v \in I, b \in B_{\varnothing}$, we have a propositional letter, $p_{v b}$. 
Axioms. (I) For distinct $b, c$ in $B_{\varnothing}$ and any $v \in I$,

$$
\sim\left(p_{v b} \wedge p_{v c}\right) \text {. }
$$

(II) For any finite $W \subseteq I$ and $h \in H_{W}$, there are only finitely many different functions $h \uparrow W$. If $\left\{h_{1}, \cdots, h_{n}\right\}$ is the set of these functions and if $W=\left\{v_{1}, \cdots, v_{k}\right\}$ and $h_{i}\left(v_{j}\right)=b_{i j}$, take as an axiom:

$$
\left(p_{v_{1} b_{11}} \wedge \cdots \wedge p_{v_{k} b_{1 k}}\right) \vee \cdots \vee\left(p_{v_{1} b_{n 1}} \wedge \cdots \wedge p_{v_{k} b_{n k}}\right) .
$$

Any finite number of these axioms is satisfiable; for if $W=\left\{v_{1}, \cdots, v_{m}\right\}$ are all the $I$-constants occurring in the given finite set of axioms and $h \in H_{W}$, let $p_{v b}$ be true if $h(v)=b$, false otherwise. Since $h \in H_{V}$, for any $V \subseteq W$, this assignment is easily seen to satisfy the given axioms.

By the compactness theorem, the whole set of axioms is satisfied. Given any interpretation satisfying the entire set of axioms, define a function as follows: for any $v \in I$, let $W=\{v\}$, then (II) asserts that $p_{v b}$ is true for at least one $b \in B$ and by $(I), p_{v b}$ is true for exactly one $b$; let $g(v)$ be the unique $b$ such that $p_{v b}$ is true. Then for any finite $W \subseteq I$ it follows using (II) that $g \nmid W$ is identical with some $h \uparrow W, h \in H_{W^{*}}$, hence $g\lceil W \subseteq h$ as required.

Theorem 1 can also be easily derived from the Tychonoff theorem for Hausdorff spaces, which is a form of the prime ideal theorem (see [6]); the proof is essentially the same as that given for Rado's lemma in [4]. Finally, for a proof of the compactness of propositional logic directly from Rado's lemma and the assumption that the set of proposition letters is well ordered, see [1].

\section{REFERENCES}

1. R. H. Cowen, A new proof of the compactness theorem for propositional logic, Notre Dame J. Formal Logic 11 (1970), 79-80. MR 43 \#4624.

2. —_ A short proof of Rado's lemma, J. Combinatorial Theory 12 (1972), 299-300.

3. N. G. de Bruijn and P. Erdös, A colour problem for infinite graphs and a problem in the theory of relations, Nederl. Akad. Wetensch. Proc. Ser. A 54=Indag. Math. 13 (1951), 369-373. MR 13, 763.

4. W. H. Gottschalk, Choice functions and Tychonoff's theorem, Proc. Amer. Math. Soc. 18 (1967), 113-115.

5. J. L. Kelly, General topology, Van Nostrand, Princeton, N.J., 1955. MR 16, 1136.

6. J. Loś and C. Ryll-Nardzewski, Effectiveness of the representation theory for Boolean algebras, Fund. Math. 41 (1954), 49-56. MR 16, 439.

7. E. Mendelson, Introduction to mathematical logic, Van Nostrand, Princeton, N.J., 1964. MR 29 \#2158.

8. J. Mycielski, Two remarks on Tychonoff's product theorem, Bull. Acad. Polon. Sci. Sér. Sci. Math. Astronom. Phys. 12 (1964), 439-441. MR 35 \#6566. 
9. A. Robinson, Introduction to model theory and to the metamathematics of algebra, North-Holland, Amsterdam, 1963. MR 27 \#3533.

10. R. Rado, Axiomatic treatment of rank in infinite sets, Canad. J. Math. 1 (1949), 337-343. MR 11, 238.

11. — A selection lemma, J. Combinatorial Theory Ser. A 10 (1971), 176-177. MR 42 \#5803.

12. E. S. Wolk, On theorems of Tychonoff, Alexander and R. Rado, Proc. Amer. Math. Soc. 18 (1967), 113-115. MR 34 \#3529.

Department of Mathematics, CunY, Queens College, Flushing, New York 11367 